$5-2018$

\title{
Nurses' Perception of the Relationship between Socioeconomic Status, Incidence Rates and Complications of a Type 2 Diabetes Diagnosis in the Adult Population
}

Malia McDowell

Dominican University of California

https://doi.org/10.33015/dominican.edu/2018.HONORS.ST.13

Survey: Let us know how this paper benefits you.

\section{Recommended Citation}

McDowell, Malia, "Nurses' Perception of the Relationship between Socioeconomic Status, Incidence Rates and Complications of a Type 2 Diabetes Diagnosis in the Adult Population" (2018). Honors Theses. 32.

https://doi.org/10.33015/dominican.edu/2018.HONORS.ST.13

This Honors Thesis is brought to you for free and open access by the Student Scholarship at Dominican Scholar. It has been accepted for inclusion in Honors Theses by an authorized administrator of Dominican Scholar. For more information, please contact

michael.pujals@dominican.edu. 


\title{
Nurses' Perception of the Relationship between Socioeconomic Status, Incidence Rates and Complications of a Type 2 Diabetes Diagnosis in the Adult Population
}

\begin{abstract}
The objective of this paper was to review the literature that reflects studies conducted on lower socioeconomic patients at risk for diabetes. These studies targeted the prevalence, medication adherence, chronic complications, and socioeconomic levels and the risk of incidence. The concluding evidence supports the research studies regarding diabetes and socioeconomic levels in that an adults' socioeconomic level has a direct negative relationship between level of finance and the overall life time risk of a new diagnosis of Type 2 diabetes and its complications such as retinopathy, neuropathy and poor clinical outcomes. Furthermore, economic status also has a direct negative correlation with increased risk of non-adherence to medications and care. The paper was supported by the Health Beliefs Model by psychologists Hochbaum, Rosenstock and Kegels (1950) that describes how the model focuses on two foundational ideas: 1) the desire to avoid illness, or conversely get well if already ill; and, 2) the belief that a specific health action will prevent, or cure, illness. The paper concludes with results on a survey of 10 nurses from the bay area and their perceptions of the relationship between low socioeconomic levels, the development of diabetes, and associated complications.
\end{abstract}

Document Type

Honors Thesis

Degree Name

Bachelor of Science

Department

Nursing

First Reader

Luanne Linnard-Palmer, EdD, RN

Second Reader

Ellen Christiansen, FNP, MSN

Keywords

DEI

Subject Categories

Nursing

This honors thesis is available at Dominican Scholar: https://scholar.dominican.edu/honors-theses/32 
Nurses' Perception of the Relationship between Socioeconomic Status, Incidence Rates and Complications of a Type 2 Diabetes Diagnosis in the Adult Population

By

Malia McDowell

Submitted in partial fulfillment of the requirements of the Nursing Department and the Honors Program

Dominican University of California

2018

First Reader: Dr. Luanne PalmerDepartment of Nursing

Second Reader: Ellen Christiansen Department of Nursing

Honors Director: Dr. Gigi Gokcek Department of Political Science/International Studies 
Nurses' Perception of the Relationship between Socioeconomic Status, Incidence Rates and Complications of a Type 2 Diabetes Diagnosis in the Adult Population

\author{
Malia McDowell
}

Dominican University of California

Spring 2018

Senior Honor Thesis

Dr. Linnard-Palmer 


\begin{abstract}
The objective of this paper was to review the literature that reflects studies conducted on lower socioeconomic patients at risk for diabetes. These studies targeted the prevalence, medication adherence, chronic complications, and socioeconomic levels and the risk of incidence. The concluding evidence supports the research studies regarding diabetes and socioeconomic levels in that an adults' socioeconomic level has a direct negative relationship between level of finance and the overall life time risk of a new diagnosis of Type 2 diabetes and its complications such as retinopathy, neuropathy and poor clinical outcomes. Furthermore, economic status also has a direct negative correlation with increased risk of non-adherence to medications and care. The paper was supported by the Health Beliefs Model by psychologists Hochbaum, Rosenstock and Kegels (1950) that describes how the model focuses on two foundational ideas: 1) the desire to avoid illness, or conversely get well if already ill; and, 2) the belief that a specific health action will prevent, or cure, illness. The paper concludes with results on a survey of 10 nurses from the bay area and their perceptions of the relationship between low socioeconomic levels, the development of diabetes, and associated complications.
\end{abstract}




\section{Acknowledgements}

I would like to thank my parents for the unconditional love and guidance they give to me. I owe all of my accomplishments and success to the emotional, mental, and financial support you continue to give. Without you, I would not have all of the opportunities I have today. Thank you for teaching me to be compassionate, hardworking, reliable, and responsible adult. I owe it all to you. 


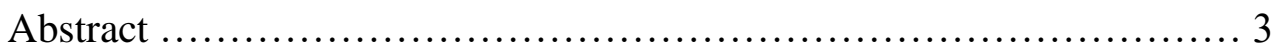

Acknowledgements .......................................................... 4

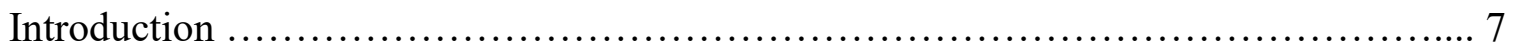

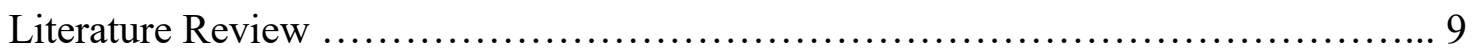

Theoretical Framework .................................................. 18

Research Design .......................................................... 19

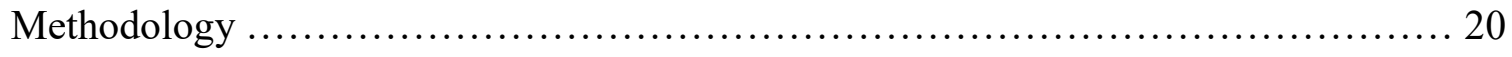

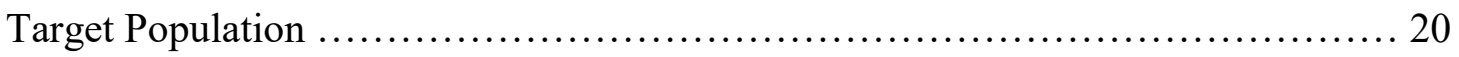

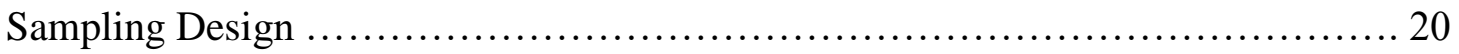

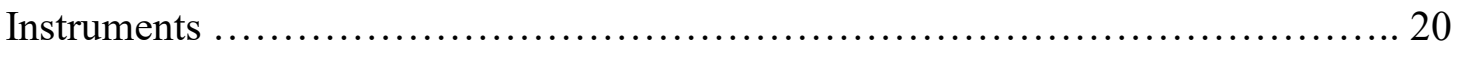

Ethical Considerations ................................................... 21

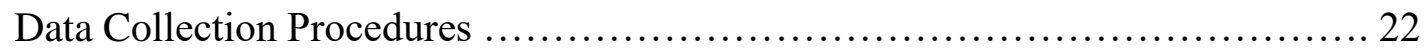

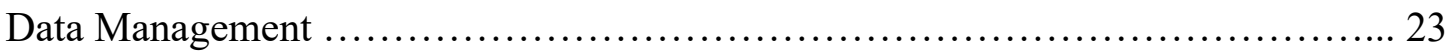

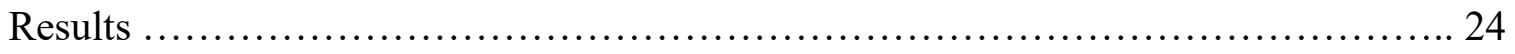

Implications for Nursing Practice...................................... 25

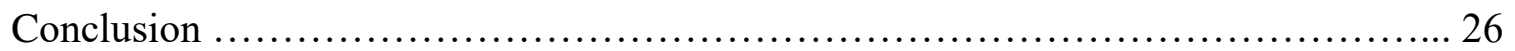

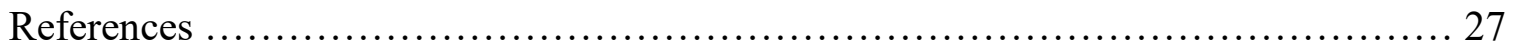


Appendix A .

Appendix B

Appendix C 


\section{Introduction}

There is a relationship between one's socioeconomic status and access to health care. Socioeconomic status is an individual or family's economic and social position in relation to others based on income, education, and occupation. Higher education levels and higher income provide that individual with better access to health care, treatment, and resources. It is important to have access to health care for promoting and maintaining health, preventing and managing disease, and reducing unnecessary disability and premature death. Without access to health care due to one's socioeconomic status, the risk of being diagnosed with type II diabetes increases.

Diabetes occurs when the body is unable to control the amount of glucose, or sugar, in the blood. When food is ingested and broken down this produces sugar, causing the amount of sugar found in the blood to rise. The liver also stores sugar, which is used when the amount of sugar in our blood is too low, for example when we're not eating. Insulin is a hormone produced by the pancreas, which helps keep the healthy amount of sugar in our blood. It can remove sugar from the blood and transport it into the cells of the body where it is needed for energy. Type 1 diabetes is a condition you are born with where the pancreas does not produce insulin. These individuals will need to take insulin shots to control their blood sugar levels. Those with type 2 diabetes still produce insulin, but their body doesn't respond to it normally. Sugar has a more difficult time entering the cells to provide energy, which keeps the blood sugar levels high. Being overweight, inactive, and having a family member with type 2 diabetes can highly increase one's chances of developing type 2 diabetes. 
Lifestyle choices can either greatly increase or decrease one's risk of developing type 2 diabetes. Living in a low socioeconomic neighborhood can decrease access to parks, libraries, and healthy grocery stores. Due to one's income and where they can afford to live limits their ability to make healthy lifestyle choices. Without access to affordable healthy food options fast food and junk food are more likely to be consumed. This can lead to increased rates of obesity. In lower socioeconomic neighborhoods they are less likely to have parks or places of recreation. This can lead to higher rates of inactivity at a younger age. Without access to health care these individuals might not be aware that obesity and inactivity greatly increase their chances of developing type 2 diabetes.

\section{Problem Statement}

Type II diabetes is a common condition that many individuals throughout the world are affected by. It is a chronic condition that affects the way the body digests sugar. According to Huether (p.462, 1996), diabetes type 2 is non-insulin dependent and insulin resistant. Insulin resistant is stated as "suboptimal response of insulin-sensitive tissues (especially liver, muscle, and adipose tissue) to insulin and is associated with obesity. Early recognition of this condition is necessary to prevent any complications that may occur. Obesity is the main reason that correlates with hyperinsulinemia and the fact that many individuals are being diagnosed with this condition. The pancreas secretes the insulin to decrease the blood sugar and with the decreased ability to do so, the patient is affected with different symptoms. Some symptoms of uncontrolled diabetes is fatigue, pruritus, recurrent infections, visual changes, or symptoms of neuropathy. The three main diagnoses of diabetes is polyuria, polydipsia, and polyphagia. Dietary measures and exercise are the main factors of prevention and treatment for Type II diabetes. 
Huether (1996) stated that type 2 diabetes mellitus is more common than type 1 and has been rising since 1940. With this finding it brings health care professionals to ask the question why? Individuals wonder if it's what people are eating and socioeconomic levels. An individual that has less money may have fewer resources. Different factors can affect this statement including lack of education, lack of resources, lack of money for healthy options, and much more. Huether (1996, pg. X) stated that a "geneticenvironmental interaction appears to be responsible for type II diabetes." The risk factors are age, obesity, hypertension, physical inactivity, and family history. This author also brings the topic of metabolic syndrome to the topic and that approximately 55 million Americans are affected by type 2 diabetes with overweight children and adolescents.

\section{Purpose Statement}

Through this research paper the author will be exploring the factors that correlate socioeconomic status and the risk of developing type two diabetes, its complications and concerns with adherence.

\section{Research Question}

How do nurses report the relationship between socioeconomic status with the risk of developing type two diabetes and associated complications?

\section{Literature Review}

\section{Introduction}

The "Correlation of Socioeconomic Status to Rates of Type 2 Diabetes Diagnosis in the Adult Population" plays a role in advancing knowledge throughout both health care 
professionals and patients. Research was found that supports diabetic management in the topics of medication adherence, the prevalence of diabetes, chronic complications, and socioeconomic status and risk of incidence. The data bases that were used were Cinahl and Iceberg.

\section{Prevalence}

Socioeconomic Status and the Prevalence of Type 2 Diabetes among Adults in Northwest China found in Diabetes Educator by Zhu, Shuzhen, 2015, aims to study the associations of socioeconomic status, lifestyle risk factors, and the prevalence of newly diagnosed type 2 diabetes in Northwest China. Based on a correlational design and random multistage sampling in communities $(\mathrm{N}=3243)$, the researchers collected data through face-to-face interviews and anthropometric measurements. In this study education, occupation, and income were considered indicators of socioeconomic status. The results of this study concluded that approximately $7.5 \%$ persons interviewed had newly diagnosed type 2 diabetes. Lifestyle risk factors, including alcohol use, unhealthy diet, and higher body mass index, were significantly positively associated with type 2 diabetes. Adjusting for demographic characteristics, hierarchical logistic regression analyses showed that unhealthy diet and high body mass index were significantly associated with type 2 diabetes. Diabetes self-management knowledge and a healthy lifestyle intervention may play a role in lowering diabetes risk in the Chinese population.

The study Detection of type 2 diabetes: what role for associated risk and protective factors and socioeconomic status found in Australian Health Review by Comino and Harris, Mark, 2012, explored associations between demographic, socioeconomic, behavioral risk, and health factors and detection of type 2 diabetes. This 
study comprises a secondary analysis of cross-sectional data collected as a part of The Australian Diabetes, Obesity and Lifestyle, also called the AusDiab study. The AusDiab study comprised a random sample drawn from 42 Census Collector Districts across Australia. Within each Census District, all homes were approached and usual residents 25 years or older were invited to participate. The study comprised a brief household interview, followed by a health check including biomedical testing at a local study center. The study found that $49.6 \%$ of participants were classified as newly diagnosed diabetics. These findings highlight the importance of systematic approaches to screening for diabetes risk focused on the whole population, with selective screening based on multifactorial assessment of diabetes risk.

In What to target and how to address in interventions for adults with low socioeconomic status found in Chronic Illness by Mayberry, Harper, \& Osborn, 2016, focuses on diabetes specific families' behaviors and its association with glycemic control and self-care. Formative research was done to inform the assessment and interventions of obstructive family behaviors like nagging, arguing, and sabotaging. The main focus was among racial/ethnic minorities and low income adults who struggle with self-care adherence. The researchers conducted a mixed methods study with adults with type 2 diabetes at a Federally Qualified Health Care Center. The participants completed a phone survey $(n=53)$ or a focus group $(n=15)$. The questions that are asked are focused on the individual's support system, how they manage diabetes, and evaluating the person that helps them the most. Out of the participants, there were 70\% African American and had low socioeconomic status. In May-August 2014 the researchers contacted 193 adults with type 2 diabetes in Nashville, Tennessee. This sample of adults with type two diabetes and 
low socioeconomic level had diverse living situations and received support most often from adult children/ grandchildren, spouses/partners, and siblings. Less than half indicated their primary support person lived with them. Family members provided diabetes-specific support most frequently with medication adherence and exercise. Adult management of type two diabetes occurs in context, which can be supportive or disruptive to self-care and glycemic control.

Social disparities in diabetes care: a general population study in Denmark found in the Scandinavian Journal of Primary Health Care by Heltberg, 2017, investigated the association between socioeconomic factors and the attainment of treatment goals and pharmacotherapy in patients with type two diabetes in Denmark. It was a cross sectional population study. The setting was the municipality of Naestved, Denmark. They studied 907 patients with type 2 diabetes identified from a random sample of 21,205 Danish citizens. The proportion of patients who were not achieving goals for diabetes care based on their HbA1c, LDL-cholesterol, blood pressure, and lifestyle, and the proportion of patients who were treated with antihypertensive and cholesterol- and glucose-lowering medication. They investigated the association of the socioeconomic factors such as age, gender, education, occupation, income, and civil status and attainment of treatment goals and pharmacotherapy in logistic regression analyses. They also investigated effect modification of cardiovascular disease and kidney disease. Results showed that middle age (40-65 years), low education level, and low household income were associated with nonattainment of goals for diabetes care. The association of socioeconomic factors with attainment of individual treatment goals varied. Patients that had low socioeconomic levels were more often obese, physically inactive, smoking, and had elevated blood 
pressure. Socioeconomic factors were not associated with treatment goals for hyperglycemia. Socioeconomic factors were inconsistently associated with pharmacotherapy. There was no difference in contacts to general practitioners according to SES. Conclusions: In a country with free access to health care, the socioeconomic factors such as middle age, low education, and low income were associated with nonattainment of goals for diabetes care.

\section{Medication Adherence}

Medication adherence is important for controlling chronic conditions, treating temporary conditions, and overall long-term health and well-being. Medication Adherence may be more Important than other Behaviours for Optimizing Glycaemic Control among LowIncome Adults found in the Journal of Clinical Pharmacy \& Therapeutics by Osborn and Mayberry (2016), sets out to identify what behaviors are most important for optimal glycemic control among low socioeconomic patients with type 2 diabetes. The study is conducted in a cross-sectional study of 314 adults with type 2 diabetes and low socioeconomic status. The Summary of Diabetes Self-Care Activities was used to assess self-care behaviors and multivariate models to test which behaviors were associated with lower $\mathrm{HbAlc}$, or better glycemic control. What this study found was that only medication adherence was significantly associated with lower $\mathrm{HbAlc}$ after adjusting for the other self-care behaviors. Medication adherence may be the most important self-care behavior for glycemic control among adults with type 2 diabetes and low socioeconomic status. In conclusion, focused efforts to improve medication adherence among low socioeconomic patient populations may improve glycemic control. 
In Continuous access to medication and health Outcomes in uninsured adults with type 2 diabetes found in Journal of the American Association of Nurse Practitioners by Toulouse and Kodadek (2016), the main focus is to have access to an affordable and consistent supply of medication. This can be challenging for the medically underserved. This article used the Social Determinants of Health framework that guided variable selection in this study, which explored the relationship between continuous access to medication and $\mathrm{HbA1c}, \mathrm{LDL}$, and $\mathrm{BP}$ in adults with type 2 diabetes who are uninsured and of low socioeconomic status. This framework is used to describe four layers of social dynamics and how it affect each other. This research purpose is focused on the achievement of recommended levels of HbA1c, low-density lipoproteins (LDLs), and blood pressure (BP) because it improves outcomes in adults with diabetes. This study was conducted using archived medical record data and a convenience sample of 65 adults. These adults received health care on a mobile health van and medications from a pharmaceutical procurement program. Pre-post data was analyzed using dependent ttests. A convenience sample was created and all MHV patients with type 2 diabetes who were 18 years or older during the study dates were considered for inclusion. Descriptive statistics were used to analyze gender, ethnicity, age, comorbid conditions, and the number of medications that each patient was prescribed. The conclusion is that access to a consistent source of medications for chronic disease is related to improved health care outcomes.

Stressors May Compromise Medication Adherence Among Adults With Diabetes and Low Socioeconomic Status found in Western Journal Of Nursing Research by Osborn, 2014, assesses the type and number of stressors they experienced in the past 
year, and the relationships between an accumulation of these stressors and self-care behaviors before and after accounting for any effects of depressive symptoms These research article was a cross-sectional study with a diverse sample of low socioeconomic status adults type two diabetes.. From June 2010 to November 2012, they enrolled 314 English and Spanish adults that were 18 years and older. These adults had a diagnosis of type two diabetes receiving outpatient care at a Federally Qualified Health Center in Nashville, Tennessee. This study examined medication adherence for type two diabetes patients. There was wide variability in the number of stressors experienced by participants: $13 \%$ reported no stressors, $47 \%$ reported 1 to 5 stressors, $30 \%$ reported 6 to 10 stressors, and $10 \%$ reported more than 10 stressors. The conclusion of the study showed that experiencing multiple chronic stressors was associated with less adherence to certain self-care behaviors. This meant less adherence to general diet and diabetes medications; and the relationship between having more stressors and less adherence to general diet varied by level of adherence.

Chronic Complications

In Association between socioeconomic status, type 2 diabetes and its chronic complications in Argentina found in Diabetes Research \& Clinical Practice by Elgart and Caporale, 2014, the researchers aim to compare the socioeconomic status of people with type 2 diabetes in Argentina with and without major chronic complications of diabetes. In this descriptive and analytic case-control study, potential candidates were identified from the electronic records of one institution of the Social Security System of the city of Córdoba. The researchers identified and recruited 387 persons each with type 2 diabetes with or without chronic complications and 774 gender- and age-matched 
persons without type 2 diabetes. Data were obtained by telephone interviews and supplemented with data from the institution's records. The results yielded persons with type 2 diabetes, particularly those with complications, reported fewer years of general education, a lower percentage of full time jobs, lower salaries and monthly household income among those with full-time jobs, and a higher propensity to spend more money than they earned. In conclusion, type 2 diabetes and the development of its complications are each positively associated with lower socioeconomic status and greater economic distress in Argentina.

The aim of the study Socioeconomic status and type 2 diabetes complications among young Adult patients in Japan found in Plos ONE by Funakoshi and Azami, 2017, the purpose was to assess the relationship between socioeconomic status and complications of type two diabetes among young adults in Japan. It is a cross sectional study. The setting was outpatient wards of 96 hospital members and clinics of Japan Federation of Democratic Medical Institutions. A total of 782 outpatients with type 2 diabetes (525 males, 257 females), aged 20-40 years as of March 31, 2012. After excluding 110 participants whose retinopathy diagnosis was in question, 672 participants were analyzed. The researchers examined the relationship between socioeconomic status and diabetes complications using a multivariate logistic regression analysis. The results showed that the prevalence of type 2 diabetic retinopathy was $23.2 \%$, while that of nephropathy was $8.9 \%$. The odds of having retinopathy were higher among junior high school graduates, patients receiving public assistance, and patients with irregular or no employment compared to those with a higher socioeconomic status. Low socioeconomic status was associated with a greater likelihood of type 2 diabetes complications in young 
adults. These findings suggest the necessity of health policies that reduce the prevalence of diabetic complications by removing any disparities.

Socioeconomic Status and Risk of Incidence

Various studies correlate socioeconomic status with being diagnosed with type 2 diabetes, but few studies examine the risk of incidence. The influence of socioeconomic status on future risk for developing Type 2 diabetes in the Canadian population between 2011 and 2022: differential associations by sex found in the International Journal for Equity in Health by Rivera and Lebenbaum, 2015, studies the future risk of being diagnosed with type 2 diabetes. Data was obtained from the Share File of the Canadian Community Health Survey (CCHS) annual component, 2011. The CCHS is a nationally representative cross sectional survey that collects information on the health status and determinants of the Canadian population. The CCHS employs a multi-stage stratified cluster design, drawing from a sample of approximately 65,000 respondents representing roughly $98 \%$ of the Canadian population, 12 years of age or older, living in the ten provinces and three territories. The researchers measured future diabetes risk using the Diabetes Population Risk Tool. The results showed that lower household income and being food insecure predicted high future diabetes risk for women, but not for men. This study contributes to the evidence informing our understanding of how socioeconomic status at the individual and ecological levels shape increased risk for diabetes.

\section{Conclusion}

These articles contribute great information to the field of nursing. The concluding evidence supports the research studies regarding diabetes and socioeconomic levels in 
that an adult's socioeconomic level has a direct negative relationship between level of finance and the overall life time risk of a new diagnosis of Type 2 diabetes and its complications. Furthermore, economic status also has a direct negative correlation with increased risk of non-adherence to medications and care. If the research was applied to daily living and patient education, there would be less incidence of type two diabetes among the adult population.

\section{Theoretical Framework}

The Health Belief model is a psychological model that focuses on attitudes and beliefs of the individual. It attempts to predict and explain health behaviors. It was first developed in the 1950s by social psychologists Hochbaum, Rosenstock and Kegels working in the U.S. Public Health Services. This model was developed in response to the failure of free Tuberculosis health screening program. The model focuses on two foundational ideas: 1.) the desire to avoid illness, or conversely get well if already ill; and, 2) the belief that a specific health action will prevent, or cure, illness.

The health belief model contributes to the topic of diabetes and socioeconomic levels by being able to target those specific environments. For example, Lo, Chair \& Lee, (2015) conducted a study with the various factors of the health belief model. They discovered that community health program "targeting this particular group should tailor strategies that can enhance individuals' self-efficacy and address barriers perceived." Multiple levels of the model can be seen throughout the articles presented through the proposed interventions and prevention strategies. With these presented it is then up to reader to decide whether the benefits are great enough to change their behavior 
if the barriers to change are not too difficult, which is represented in the last level of the health belief model.

The health belief model can help nursing care by preventing any illnesses and taking actions on the illness. The American Diabetes Association conducted a study of the relationship between the Health Belief Model and Compliance of Persons with Diabetes Mellitus (Cerkoney, 2017). Thirty insulin-treated diabetic individuals were interviewed in their homes 6-12 months after having attended diabetic education classes at a community hospital. This shows an example of how education can be utilized throughout a community. The nurse has the role of an advocate and education is a top priority for them. This model keeps the nurse thinking "what should this patient focus on preventing depending on what they are at risk for.

\section{Methods Section}

The purpose of this study was to explore nurse's perceptions on how socioeconomic status influences the risk of developing type two diabetes and contributes to the development of complications.

\section{Target Population}

The target population was nurses in the bay area who work with a population newly diagnosed adult patients who have a new incidence of diabetes type 2 .

\section{Subjects}

The subjects were be Dominican alumni, Rho Alpha society members, and Dominican Nursing faculty, as well as any other eligible subjects. 


\section{Sample size}

The sample size is 10 including a variation nurses in a variety of health care settings.

\section{Sampling procedures}

The sampling procedure will include purposeful (non-probability) sampling and snowball sampling. Snowballing will be done through handing out the surveys to nursing professors and them distributing it to their nursing colleagues.

\section{Instrument}

The instrument that is used for this study will be developed by the authors as a survey to assess socioeconomic levels with diabetic patients and reports of experiences of low economic status with the development of complications of a newly diagnosed adults type 2 diabetic. These surveys will be handed out to other nurses including Dominican alumni, Rho Alpha society, and Dominican Nursing faculty. This will be distributed by the purposeful sampling and snowball technique.

\section{Survey}

Demographics- ( )

Age- ( )

$\operatorname{Sex}^{-}(\mathbf{)}$

Job title- ( )

Ethnicity- ( )

Nurse's Socioeconomic level- low, middle or high 
City that you live in:

City that you work in:

Type of hospital or health care setting:

Length of time in nursing:

Educational preparation:

Questions:

- $\quad$ Do you care for diabetic patients, if no, thank you and do not continue.

- What are your perceptions on socioeconomic status and the risk of diabetes?

- What is your prevention education that you provide to patients?

- What do you think causes a low socioeconomic patient to be more at risk for diabetes and how can we as nurses prevent that?

- What resources do you provide to patients that are low socioeconomic class and are not able to afford basic resources?

- How do you screen for patients with diabetes?

- What education or tools do you provide to patients that are going to be discharged with diabetes?

\section{Reliability}


In this pilot study, reliability measurement was not feasible.

\section{Validity}

The study demonstrated content and face validity by the author showing the instrument to two doctoral prepared nursing professors and soliciting feedback on the relationship between the overall research question guiding the study and the survey.

\section{Alpha level/effect size:}

Effect size computation was not feasible in this small pilot study.

\section{Step by step procedures to collect data}

For the study, data was collected by:

- $\quad$ Secured IRB Approval

- Secured content and face validity of instruments by talking to two doctoral prepared Nursing professors at Dominican.

- $\quad$ Clarified the specific goals in order to solve the problem. With this study, the goal is to determine the influence of socioeconomic status with diabetic patients.

- $\quad$ Planed an approach and methods, such as distinguishing the target population and location of where the data will be gathered.

- $\quad$ Requested permission from the university to send out an electronic and paper copies of the survey to alumni, Rho Alpha members and current and past faculty. 
- Disseminatee the instrument using purposeful and snowball

sampling

- Collected data via the author-created instrument and analyzee data using descriptive statistics.

- $\quad$ Established efficient results from the descriptive statistics and surveys used for the study.

- Established an overview, evaluation, and conclusion of the study if the goals have been met to solve the problem.

* Submitted an abstract for the Dominican University of California Scholarly and Creative Works conference and was accepted. The oral presentation took place Thursday April 19 $9^{\text {th }}, 2018$

\section{Results}

The average age was 50 years old. The practice specialty areas included Case Manager, PICU, Pediatrics, Adult Medical Surgical, and Nursing education.

1. $9 / 10$ subjects described that there is a relationship between economic status and the risk for developing diabetes.

2. $9 / 10$ subjects agreed that there is a direct correlation with economic status and development of complications.

3. All but one participant said that an education program on prevention should focus on diet. 
4. Participants described the need for referral to support groups, social workers, and appropriate handouts.

5. Participants agreed that screening should include economic status so that needs are identified early.

6. Participants described that educational tools used include pink pants, expanded teaching sessions, and a variety of materials.

7. The last question in the survey asked participants about improving undergraduate education. Suggestions made included: Advanced Pathophysiology, Advanced Pharmacology, Advanced Nutrition, Case Studies, interviews with diabetic nurses, shadowing a diabetic educator, and ensuring that every student in nursing school cares for diabetic patients.

\section{Implications for nursing practice:}

Implications for this study are to acknowledge the correlation between socioeconomic status and the risk of developing type 2 diabetes. Having a lower socioeconomic status can correlate with having poor lifestyle choices such as poor nutrition intake, lack of exercise, and poor medication adherence. It is important for nurses to know that their patients may be at risk for developing diabetes in order to give proper teaching, education, and resources to their patients.

Suggestions for further research:

This pilot study has shown that further education is needed on the connection between type $2 \mathrm{DM}$ and LSES. The literature showed that awareness of prevention of DM is 
needed, especially for those in LSES. Screenings continues to be vitally important in the early identification of type $2 \mathrm{dm}$. More literature is needed that provides basic instructions for those with lower literacy levels (visual imagery).

\section{References}

Cerkoney, K. (2017). The relationship between the health belief model and compliance of persons with diabetes mellitus.vol. 3 no. 5 594-598, 1.

doi:https://doi.org/10.2337/diacare.3.5.594

Comino, E. J., Harris, M. F., Shaw, J. E., \& Jayasinghe, U. W. (2012). Detection of type

2 diabetes: what role for associated risk and protective factors and socioeconomic status? Australian Health Review, 36(3), 349. doi:10.1071/ah11047

Elgart, J. F., Caporale, J. E., Asteazarán, S., Fuente, J. L., Camilluci, C., Brown, J. B., . . . Gagliardino, J. J. (2014). Association between socioeconomic status, type 2 diabetes and its chronic complications in Argentina. Diabetes Research and Clinical Practice, 104(2), 241-247. doi:10.1016/j.diabres.2014.02.010

Funakoshi, M., Azami, Y., Matsumoto, H., Ikota, A., Ito, K., Okimoto, H., \& ... Miura, J. (2017). Socioeconomic status and type 2 diabetes complications among young Adult patients in Japan. Plos ONE, 12(4), 1-14. doi:10.1371/journal.pone.0176087 Heltberg, A., Andersen, J. S., Kragstrup, J., Siersma, V., Sandholdt, H., \& Ellervik, C. (2017).Social disparities in diabetes care: a general population study in Denmark. Scandinavian Journal Of Primary Health Care, 35(1), 54-63. doi:10.1080/02813432.2017.1288702 
Lo, S. S., Chair, S. Y., \& Lee, F. K. (2015). Original Article: Factors associated with health-promoting behavior of people with or at high risk of metabolic syndrome: Based on the health belief model. Applied Nursing Research, 28197-201.

doi:10.1016/j.apnr.2014.11.001

Mayberry, L. S., Harper, K. J., \& Osborn, C. Y. (2016). Family behaviors and type 2 diabetes: What to target and how to address in interventions for adults with low socioeconomic status. Chronic Illness, 12(3), 199-215. doi:10.1177/1742395316644303

Osborn, C. Y., Mayberry, L. S., \& Kim, J. M. (2016). Medication adherence may bemore important than other behaviours for optimizing glycaemic control among low-income adults. Journal of Clinical Pharmacy and Therapeutics, 41(3), 256-259. doi:10.1111/jcpt.12360

Osborn, C. Y., Mayberry, L. S., Wagner, J. A., \& Welch, G. W. (2014). Stressors May Compromise Medication Adherence Among Adults With Diabetes and Low Socioeconomic Status. Western Journal Of Nursing Research, 36(9), 1091-1110. doi: $10.1177 / 0193945914524639$

Rivera, L. A., Lebenbaum, M., \& Rosella, L. C. (2015). The influence of socioeconomic status on future risk for developing Type 2 diabetes in the Canadian population between 2011 and 2022: differential associations by sex. International Journal for Equity in Health, 14(1). doi:10.1186/s12939-015-0245-0

Toulouse, C., \& Kodadek, M. (2016). Continuous access to medication and health 
Outcomes in uninsured adults with type 2 diabetes. Journal Of The American Association Of Nurse Practitioners, 28(6), 327-334. doi:10.1002/2327-6924.12326

Zhu, S., Hu, J., Mccoy, T. P., Li, G., Zhu, J., Lei, M., . . Kong, L. (2015).

Socioeconomic Status and the Prevalence of Type 2 Diabetes Among Adults in Northwest China. The Diabetes Educator, 41(5), 599-608.

doi:10.1177/0145721715598382 


\section{Appendix A}

\section{Demographics}

Age- ( )

Gender- ( )

Job title- ( )

Ethnicity- ( )

Socioeconomic level- low, middle or high: ( )

City that you live in:

City that you currently work in:

Specialty area:

Are you a DM educator: Yes No

Do you work directly with patients who have new cases of DM? Yes No

Questions:

1. Do you think there is a direct correlation between socioeconomic status and the risk or prevalence of developing diabetes? If yes, please describe.

2. Do you think there is a direct correlation between socioeconomic status and the development of type 2 complications? If yes, please describe.

3. What do you think causes a low socioeconomic patient to be more at risk for diabetes and how can we as nurses prevent that? 
4. Describe how you implement a prevention education program towards the complications associated with type 2 DM:

5. What resources do you provide to patients that are low socioeconomic class and are not able to afford basic resources?

6. How do you screen for economic status in patients with diabetes?

7. What education or tools do you provide to patients that are discharging with diabetes?

8. What suggestions do you have for undergraduate nursing education to promote a better understanding of the relationship between socioeconomic status and the prevalence of type $2 \mathrm{DM}$ and its associated complications? 


\section{Appendix B}

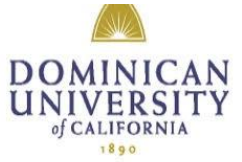

March 15, 2018

Malia McDowell

50 Acacia Ave.

San Rafael, CA 94901

Dear Malia:

I have reviewed your proposal entitled An Investigation into Nurse's Perception of the Correlation of Socioeconomic Status on Rates of Type 2 Diabetes Diagnosis in the Adult Population submitted to the Dominican University Institutional Review Board for the Protection of Human Participants (IRBPHP Application, \#10686). I am approving it as having met the requirements for minimizing risk and protecting the rights of the participants in your research.

In your final report or paper please indicate that your project was approved by the IRBPHP and indicate the identification number.

I wish you well in your very interesting research effort.

Sincerely,

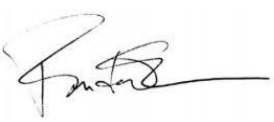

Randall Hall, Ph.D.

Chair, IRBPHP

Cc: Luanne Linnard-Palmer

Institutional Review Board for the Protection of Human Participants 


\section{Appendix C}

\section{DOMINICAN UNIVERSITY OF CALIFORNIA}

Consent form for participation in the study titled, "An Investigation in to Nurse's Perception of the Correlation of Socioeconomic Status on Rates of Type 2 Diabetes Diagnosis in the Adult Population"

1. I understand that I am being asked to participate as a Participant in a research study designed to assess nurses' perceptions of the relationship (correlation) between socioeconomic status and the development and complications associated with type 2

Diabetes Mellitus. This research is part of Malia Senior Thesis research project at Dominican University of California, California. This research project is being supervised by Dr. Luanne Linnard-Palmer, Dominican University of California Nursing Professor.

2. I understand that participation in this research will involve taking part in a written or oral short interview (20-30 minutes), which will include perceptions and experiences between Diabetes Mellitus and socioeconomic levels of patients.

3. I understand that my participation in this study is completely voluntary and I am free to withdraw my participation at any time.

4. I have been made aware that this is a confidential survey. All personal references and identifying information will be eliminated and all participants will be identified by numerical code only; the master list for these codes will be kept by the student in a locked file, separate from the survey. One year after the completion of the research, all written and recorded materials will be destroyed. 
5. I am aware that all study participants will be furnished with a written summary of the relevant findings and conclusions of this project. Such results will not be available until May 1, 2018.

6. I understand that I will be discussing topics of professional and personal perceptions. And that I may refuse to answer any question that causes me distress or seems an invasion of my privacy. I may elect to stop the interview at any time.

7. I understand that my participation involves no physical risk, but may involve some psychological discomfort, given the nature of the topic being addressed in the interview. If I experience any problems or serious distress due to my participation, Malia will provide, at no cost to me, a one-time consultation with a licensed therapist. The student may be contacted (Malia McDowell, malia.mcdowell@students.dominican.edu).

8. I understand that if I have any further questions about the study, I may contact the student at [malia.mcdowell@students.dominican.edu] or her research supervisor, [Dr. Luanne Linnard-Palmer at linnard@dominican.edu], If I have further questions or comments about participation in this study, I may contact the Dominican University of California Institutional Review Board for the Protection of Human Participants (IRBPHP), which is concerned with the protection of volunteers in research projects. I may reach the IRBPHP Office by calling (415) 482-3547 and leaving a voicemail message, by FAX at (415) 257-0165 or by writing to the IRBPHP, Office of the Associate Vice President for Academic Affairs, Dominican University of California, 50 Acacia Avenue, San Rafael, CA 94901. 
9. All procedures related to this research project have been satisfactorily explained to me prior to my voluntary election to participate. I HAVE READ AND UNDERSTAND ALL OF THE ABOVE EXPLANATION REGARDING THIS STUDY. I VOLUNTARILY GIVE MY CONSENT TO PARTICIPATE. A COPY OF THIS FORM HAS BEEN GIVEN TO ME FOR MY FUTURE REFERENCE. 